\title{
Solving for closure errors due to polarization leakage in radio interferometry of unpolarized sources
}

\author{
S. Bhatnagar and R. Nityananda
}

\author{
National Centre for Radio Astrophysics (TIFR), Post Bag 3, Ganeshkhind, Pune 411 007, India \\ e-mail: sanjay@ncra.tifr.res.in, rajaram@ncra.tifr.res.in \\ Received 10 April 2001 / Accepted 30 May 2001
}

\begin{abstract}
Mechanical and electronic imperfections can result into polarization leakage in individual antennas of a radio interferometer. Such leakages manifest themselves as closure errors even in co-polar visibility measurements of unpolarized sources. This paper describes and tests a method for the study of polarization leakage for radio interferometric telescopes using only the nominally co-polar visibilities for unpolarized calibrators. Interpretation of the resulting closure phases on the Poincaré sphere is presented. Since unpolarized sources are used, the actual solutions for leakage parameters is subject to a degeneracy which is discussed. This however, does not affect the correction of closure errors in our scheme.
\end{abstract}

Key words. methods: data analysis - techniques: polarimetric - techniques: interferometric

\section{Introduction}

The mutual coherence function (also called the visibility function) for an unresolved and unpolarized source, measured by an interferometer array can be modeled as a product of antenna based complex gains. These complex gains can be derived from the measured visibility function using the standard algorithm, which we call antsol. antsol forms the central engine of most amplitude and phase calibration schemes used for radio interferometric data. (The earliest published reference for an algorithm for antsol of which we are aware is Thompson \& D'Addario 1982.)

Usually one measures the components of the incident radiation along two orthogonal polarization states by using two separate feeds. The signals from the two feeds travel through essentially independent paths till the correlator. However, due to mechanical imperfections in the feed or imperfections in the electronics, the two signals can leak into each other at various points in the signal chain.

At the correlator, signals from all the antennas are multiplied with each other and the results averaged to produce the visibilities. The signals of same polarization are multiplied to produce the co-polar visibilities while the signals of orthogonal polarizations are multiplied to produce the cross-polar visibilities. The co-polar and crosspolar visibilities can be used to compute the full Stokes visibility function. Antenna based instrumental polarization and polarization leakage can be derived from the full

Send offprint requests to: S. Bhatnagar,

e-mail: sanjay@ncra.tifr.res.in
Stokes coherence function for a source of known structure (usually an unresolved source) (Hamaker et al. 1996; Sault et al. 1996, henceforth HBS).

The correlator used for the Giant Metrewave Radio Telescope (GMRT) by default computes the co-polar visibilities using the Indian mode of the VLBA Multiplier and Accumulator (MAC) chip. Here we describe a method, which we call leaky antsol, for the computation of the leakages using only the co-polar visibility function for an unpolarized source. Following the notation used by HBS, we label the two orthogonal polarizations by $p$ and $q$ to remind us that the formulation is independent of the precise orthogonal pair of polarization states chosen.

Section 2 describes the motivation which led to this analysis. For orientation, Sect. 3 starts with the problem of solving for the usual complex antenna based gains and sets up an iterative scheme for the solution. The problem of simultaneously solving for the complex antenna gains and leakages is then posed in Sect. 3.1 and a similar iterative scheme is set up. Section 3.2 presents the results of the simulations done to test the scheme. Section 4.1 presents some results using the GMRT at $150 \mathrm{MHz}$. Also, we were fortunate to have the $L$-band feeds of one of the GMRT antennas converted from linear to circular polarization. We observed 3C147 in this mode where all baselines with this special antenna measured the correlation between nominally linear and circular polarization. Results of this experiment demonstrate that the leakage solutions are indeed giving information about the polarization properties of the feeds. These results and their interpretation on the Poincaré sphere are presented in Sect. 4.2. Section 5 gives the interpretation of the leakage solutions and 
discusses closure errors due to polarization leakage using the Poincaré sphere.

\section{Motivation}

Rogers (1983) pointed out in the context of the VLBA, that the polarization leakage cause closure errors even in nominally co-polar visibilities. Massi et al. (1997) have carried out a detailed study of this effect for the telescopes of the European VLBI Network (EVN). Our motivation in this paper is that the current single sideband GMRT correlator uses the so called Indian mode of the VLBA MAC chips to produce only the co-polar visibilities. Also, the planned Walsh switching has not yet been implemented at the GMRT and in any case, would not eliminate leakage generated before the switching point. Tests done using strong point source dominated fields show unaccounted closure errors at a few percent level. The motivation behind developing an algorithm to solve for gains and leakages simultaneously, using only the co-polar visibilities was to determine if the measured closure errors could be due to polarization leakage in the system. Estimates of leakage can then be used in the primary calibration to remove the effects of polarization leakage. This is where this paper differs from the earlier work of HBS which is about the calibration using the full Stokes visibility function, needed for observations of polarized sources. The polarization leakage in some of the EVN antennas corrupts the co-polar visibilities at a level visible as a reduction in the dynamic range of the maps (Massi \& Aaron 1997a,b; Massi et al. 1998). Thus such a method can also be used in imaging data from the EVN and other telescopes affected by such closure errors.

Let $g_{i}^{p}$ represent the complex gain for the $p$ polarization channel of the $i$ th antenna and $\alpha_{i}^{q}$ represent the leakage of the $q$-polarization signal into the $p$-polarization channel. The electric field measured by antenna $i$ can then be written as

$E_{i}^{p}=g_{i}^{p} E_{i, \circ}^{p}+\alpha_{i}^{q} E_{i, \circ}^{q}$

where $E_{i, \circ}^{p}$ and $E_{i, \circ}^{q}$ are the responses of an ideal antenna to the incident radiation in the $p$ - and $q$-polarization states respectively ${ }^{1}$. For an unpolarized source of radiation, $\left\langle E_{i, 0}^{p} E_{j, 0}^{q \star}\right\rangle=0$. The co-polar visibility for such a source, measured by an interferometer using two antennas denoted by the subscripts $i$ and $j$, is given by

$\rho_{i j}^{p p}=g_{i}^{p} g_{j}^{p \star} \rho_{i j, \circ}^{p p}+\alpha_{i}^{q} \alpha_{j}^{q \star} \rho_{i j, \circ}^{q q}+\epsilon_{i j}$

where $\epsilon_{i j}$ is independent Gaussian random baseline based noise and $\rho_{i j, \circ}^{p p}=\left\langle E_{i, 0}^{p} E_{j, 0}^{p \star}\right\rangle$ and $\rho_{i j, \circ}^{q q}=\left\langle E_{i, \circ}^{q} E_{j, 0}^{q \star}\right\rangle$ are the two ideal co-polar visibilities. $\epsilon_{i j}$ usually represents the contribution to $\rho_{i j}^{p p}$ which cannot be separated into

1 Note that Eq. (1) is equivalent to the Eq. (1) of Massi et al. (1997), who use a different parameterization. Strictly speaking, the ratio $\alpha_{i}^{q} / g_{i}^{p}$ is the correct measure of the leakage and this is what has been plotted in Fig. 3 below. This does not however affect the computational scheme described here. antenna based quantities. $\epsilon_{i j}$ therefore is a measure of the intrinsic closure errors in the system and is usually small.

For an unpolarized point source $\left\langle E_{i, 0}^{p} E_{j, 0}^{p \star}\right\rangle=$ $\left\langle E_{i, \circ}^{q} E_{j, \circ}^{q \star}\right\rangle=\rho_{i j, \circ}^{p p}=I / 2$ where $I$ is the total flux density. Writing $X_{i j}^{p p}=\rho_{i j}^{p p} / \rho_{i j, \circ}^{p p}$ we get

$X_{i j}^{p p}=g_{i}^{p} g_{j}^{p \star}+\alpha_{i}^{q} \alpha_{j}^{q \star}+\epsilon_{i j}$

where $\epsilon_{i j}$ now refers to the baseline based noise in $X_{i j}^{p p}$.

Assuming $\alpha_{i}^{q}$ s to be negligible, the usual antsol algorithm estimates $g_{i}^{p}$ s such that $\sum_{\substack{i, j \\ i \neq j}}\left|X_{i j}^{p p}-g_{i}^{p} g_{j}^{p \star}\right|^{2}$ is minimized (see Sect. 3). Normally, Walsh switching (Thompson et al. 1986) is used to eliminate the polarization leakage due to cross-talk between the signal paths, such that $\alpha_{i}^{q} \alpha_{j}^{q \star} \ll \epsilon_{i j}$. However, $\alpha_{i}^{q}$ s can also be finite due to mechanical imperfections in the feed or the crosspolar primary beam, which cannot be eliminated by Walsh switching.

In the case of significant antenna based polarization leakage (compared to $\sqrt{\epsilon_{i j}}$ ), the second term in Eq. (3) involving $\alpha_{i}^{q} \mathrm{~S}$ will combine with the closure noise $\epsilon_{i j}$. The polarization leakage therefore manifests itself as increased closure errors (see Sect. 5 for a geometric explanation on the Poincaré sphere).

\section{Algorithm and simulation}

In the absence of any polarization leakage, $g_{i}$ s can be estimated by minimizing

$S=\sum_{\substack{i, j \\ i \neq j}}\left|X_{i j}^{p p}-g_{i}^{p} g_{j}^{p \star}\right|^{2} w_{i j}^{p p}$

with respect to $g_{i} \mathrm{~s}$, where $w_{i j}^{p p}=1 / \sigma_{i j}^{2}, \sigma_{i j}$ being the variance on the measurement of $X_{i j}^{p p}$.

In Eq. (2), if $\rho_{i j, \circ}^{p p}$ accurately represents the source structure, $X_{i j}^{p p}$ will have no source structure dependent terms and is purely a product of two antenna dependent complex gains. For a resolved source, $\rho_{i j, o}^{p p}$ can be estimated from the image of the source.

Evaluating $\frac{\partial S}{\partial g_{i}^{p \times *}}$ and equating it to zero ${ }^{2}$ (Bhatnagar 1999), we get

$g_{i}^{p}=\frac{\sum_{\substack{j \\ j \neq i}} X_{i j}^{p p} g_{j}^{p} w_{i j}^{p p}}{\sum_{\substack{j \\ j \neq i}}\left|g_{j}^{p}\right|^{2} w_{i j}^{p p}}$.

This can also be derived by equating the partial derivatives of $S$ with respect to real and imaginary parts of $g_{i}^{p \star}$.

Since the antenna dependent complex gains also appear on the right-hand side of Eq. (5), it has to be solved iteratively starting with some initial guess for $g_{j}$ s or

\footnotetext{
${ }^{2}$ Complex derivatives can be evaluated by treating $g_{i}^{p}$ and $g_{i}^{p \star}$ as independent variables (Palka 1990).
} 
initializing them all to 1 . Equation (5) can be written in the iterative form as:

$g_{i}^{p, n}=g_{i}^{p, n-1}+\lambda\left[g_{i}^{p, n-1}-\frac{\sum_{\substack{j \\ j \neq i}} X_{i j}^{p p} g_{j}^{p, n-1} w_{i j}^{p p}}{\sum_{\substack{j \\ j \neq i}}\left|g_{j}^{p, n-1}\right|^{2} w_{i j}^{p p}}\right]$

where $n$ is the iteration number and $0<\lambda<1$. Convergence would be defined by the constraint $\left|S_{n}-S_{n-1}\right|<\beta$ (the change in $S$ from one iteration to another) where, $\beta$ is the tolerance limit and must be related to the average value of $\epsilon_{i j}$. Equation (6) forms the central engine for the classical antsol algorithm used for primary calibration of the visibilities and in self-calibration for imaging purposes. This algorithm was suggested by Thompson \& D'Addario (1982).

\subsection{The leaky antsol}

In the presence of significant polarization leakage, Eq. (3) can be used to re-write Eq. (4) as

$S=\sum_{\substack{i, j \\ i \neq j}}\left|X_{i j}^{p p}-\left(g_{i}^{p} g_{j}^{p^{\star}}+\alpha_{i}^{q} \alpha_{j}^{q^{\star}}\right)\right|^{2} w_{i j}^{p p}$.

In this form, $S$ is an estimator for the true closure noise $\epsilon_{i j}$ rather than the artificially increased closure noise $\left(\alpha_{i}^{q} \alpha_{j}^{q \star}+\epsilon_{i j}\right)$ due to the presence of polarization leakage. get

Equating the partial derivatives $\frac{\partial S}{\partial g_{i}^{p \star}}, \frac{\partial S}{\partial \alpha_{i}^{q \star}}$ to zero, we

$$
\begin{gathered}
g_{i}^{p}=\frac{\sum_{\substack{j \\
j \neq i}} X_{i j}^{p p} g_{j}^{p} w_{i j}^{p p}-\alpha_{i}^{q} \sum_{\substack{j \\
j \neq i}} \alpha_{j}^{q \star} g_{j}^{p} w_{i j}^{p p}}{\sum_{\substack{j \\
j \neq i}}\left|g_{j}^{p}\right|^{2} w_{i j}^{p p}} \\
\alpha_{i}^{q}=\frac{\sum_{\substack{j \\
j \neq i}} X_{i j}^{p p} \alpha_{j}^{q} w_{i j}^{p p}-g_{i}^{p} \sum_{\substack{j \\
j \neq i}} g_{j}^{p \star} \alpha_{j}^{q} w_{i j}^{p p}}{\sum_{\substack{j \\
j \neq i}}\left|\alpha_{j}^{q}\right|^{2} w_{i j}^{p p}} .
\end{gathered}
$$

These non-linear equations can also be iteratively solved.

Equation (3), which expresses the observed visibilities on a point source unpolarized calibrator in terms of the gains and leakage coefficients of the antennas, would take the same form if written in an arbitrary orthogonal basis. It is clear that the $g$ 's and the $\alpha$ 's will change when we change the basis, so this means that the equations cannot have a unique solution. This situation is familiar from ordinary self-calibration, when only relative phases of antennas are determinate, with one antenna acting as an arbitrary reference. For observations of unpolarized sources, we can similarly say that any feed can be chosen as a reference polarization, with zero leakage, and other feeds have gains and leakages in the basis defined by this reference. Other conventions may be more convenient, as discussed in the appendix which discusses degeneracy in detail.

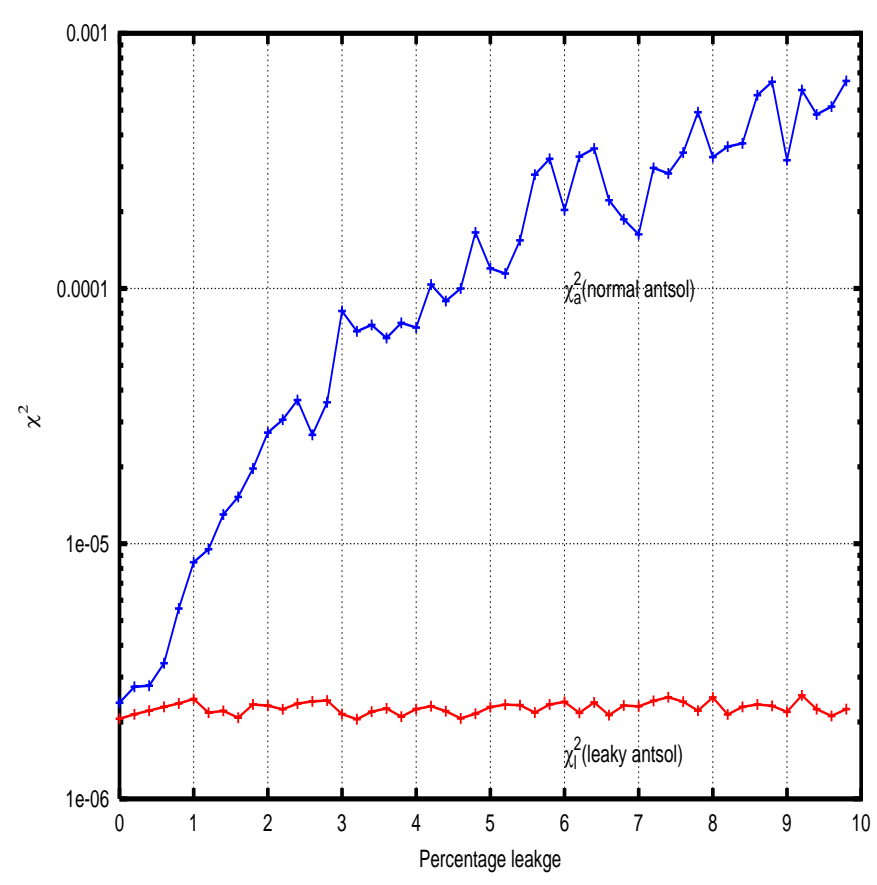

Fig. 1. Figure showing the results of the simulations. The top curve is the value of $\chi^{2}$ using the classical antsol $\left(\chi_{\mathrm{a}}^{2}\right)$. The bottom curve is the value of $\chi^{2}$ using the leaky antsol $\left(\chi_{1}^{2}\right)$ as a function of the percentage polarization leakage.

\subsection{Results of the simulations}

We simulated visibilities with varying fraction of polarization leakage in the antennas to test the algorithm as follows. The antenna based signal and leakage were constructed as $g_{i}=R_{\mathrm{g}}$ and $\alpha_{i}=f \cdot R_{\alpha}$ where $R_{\mathrm{g}}$ and $R_{\alpha}$ were drawn from the same Gaussian random population. The visibility from two antennas $i$ and $j$ was then constructed as $X_{i j}=g_{i} g_{j}^{\star}+\alpha_{i} \alpha_{j}^{\star}+\epsilon_{i j}$ for $0 \leq f<0.1$. This is equivalent to a visibility of an unpolarized point source of unit strength with a complex antenna based gain $g_{i}$ and leakage $\alpha_{i}$ of strength proportional to $f$. Equation (6) was then used to compute $g_{i}$ and residual $\chi^{2}$ computed as $\chi_{\mathrm{a}}^{2}=\sum_{i j}\left|1-\frac{X_{i j}}{g_{i} g_{j}^{\star}}\right|^{2}$. The computed values of $g_{i}^{p}$ were then used to compute improved estimates for $g_{i}^{p}$ by simultaneously solving for $g_{i}^{p}$ and $\alpha_{i}^{q}$ using the iterative forms of Eqs. (8) and (9). The derived values of $g_{i}^{p}$ and $\alpha_{i}^{q}$ matched the true values to within the tolerance limit. A new $\chi^{2}$ was computed as $\chi_{1}^{2}=\sum_{i j}\left|1-\frac{X_{i j}}{\left(g_{i} g_{j}^{\star}+\alpha_{i} \alpha_{j}^{\star}\right)}\right|^{2}$. The values of $\chi_{\mathrm{a}}^{2}$ and $\chi_{\mathrm{l}}^{2}$ as a function of $f$ are plotted in Fig. 1. The two curves become distinguishable when the leakage is significantly greater than $\epsilon_{i j}$ (for $f$ greater than $\sim 1 \%$ ). After that, the value of $\chi_{1}^{2}$ is consistently lower than $\chi_{a}^{2}$, where the contribution of antenna based leakage has not been removed. Also notice that $\chi_{1}^{2}$ remains constant while $\chi_{\mathrm{a}}^{2}$ quadratically increases as a function of $f$. This is due to the fact that antsol treats the antenna based polarization leakage as closure errors resulting in an increased $\chi^{2}$ with increasing fractional leakage. 


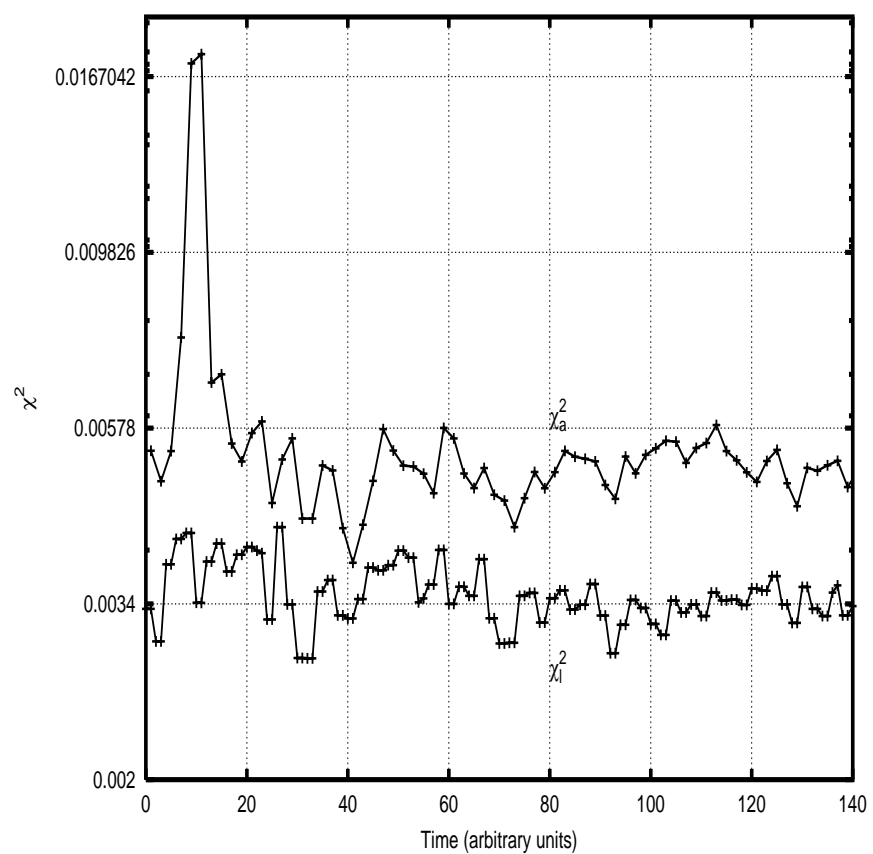

Fig. 2. Figure showing the results using GMRT data at $150 \mathrm{MHz}$ for the compact Galactic plane source 1830-36. The top curve is the value of $\chi^{2}$ using the classical antsol $\left(\chi_{\mathrm{a}}^{2}\right)$. The bottom curve is the value of $\chi^{2}$ using the leaky antsol $\left(\chi_{1}^{2}\right)$ as a function of time.

\section{Real data}

\section{1. $150 \mathrm{MHz}$ data}

Engineering measurements for polarization isolation at $150 \mathrm{MHz}$ for the GMRT show significant polarization leakage in the system. We therefore used leaky antsol to calibrate the data from the Galactic plane phase calibrator 1830-36 which is known to be less than $0.2 \%$ polarized at $1.4 \mathrm{GHz}$. The percentage polarization at $150 \mathrm{MHz}$ is not known, but it is expected to decrease further and it was taken to be an unpolarized point source.

Fractional polarization leakage $\left(\left|\alpha_{i}^{q} / g_{i}^{p}\right|\right)$ of up to $100 \%$ was measured for most of the antennas, which is consistent with the estimated leakage measured from system engineering tests. Again, $\chi_{\mathrm{a}}^{2}$ and $\chi_{1}^{2}$ were computed and the results are shown in Fig. 2. The 150-MHz GMRT band suffers from severe radio frequency interference (RFI). The sharp rise in the value of $\chi_{\mathrm{a}}^{2}$ around sample number 10 is due to one such RFI spike. This spike is present in the total power data from all antennas at this time. On an average, the $\chi^{2}$ reduces by $\sim 60 \%$ when leakage calibration is applied $\left(\chi_{1}^{2}\right)$. This is consistent with polarization leakage being a major source of non-closure at this frequency.

\subsection{L-band data with circular feed}

The GMRT $L$-band feeds are linearly polarized. For the purpose of a VLBI experiment conducted in December 2000, the $L$-band feed of one of the antennas was converted to a circularly polarized feed. The rest of the
$L$-band feeds were linearly polarized and we took this opportunity to measure correlations between the circularly polarized antenna with other linearly polarized antennas using the source $3 \mathrm{C} 147$. Two scans of approximately one hour long observations were done using the single side band GMRT correlator. This correlator computes only co-polar visibilities. With this configuration of feeds, visibilities between the circularly polarized antenna and all other linearly polarized antennas corresponds to correlation between the nominal $X$ - and $R$-polarizations, labeled by $R X$, were recorded in the first scan. The polarization of the circularly polarized antenna was then flipped for the second scan to record the correlation between the nominal $X$ - and $L$-polarization states, labeled by $L X$.

The VLA Calibrator Manual ${ }^{3}$ lists the percentage polarization $\left(\frac{\sqrt{Q^{2}+U^{2}+V^{2}}}{I}\right)$ for 3 C147 at $L$-band $<0.1 \%$. The cross-polar terms in Eq. (2), which are assumed to be zero, will therefore contribute an error of the order of $0.1 \%$. These cross-polar terms will be, however, multiplied by gains of type $g_{i}^{p} \alpha_{i}^{q \star}$. Since $g_{i}^{p}$ and $\alpha_{i}^{q}$ are both assumed to be uncorrelated between antennas, this error will manifest as random noise in Eq. (3). Within the limits of other sources of errors, the source $3 \mathrm{C} 147$ can therefore be considered to be a completely unpolarized source.

\subsubsection{Results and interpretation}

The response of an ideal circularly polarized antenna to unpolarized incident radiation can be expressed as a superposition of two linear polarization states as $E_{i, \mathrm{o}}^{R}=$ $E_{i, \circ}^{X} \mathrm{e}^{\iota \delta}+E_{i, \circ}^{Y} \mathrm{e}^{-\iota \delta}$ where, the superscripts $R, X$ and $Y$ denote the right circular and the two linear polarization states respectively. $\delta$ is half the phase difference between the two linear polarization states and is equal to $\pi / 4$ for right-circular polarization and $-\pi / 4$ for left-circular polarization. Writing the general Eq. (1) for right-circularly polarized antenna as $E_{i}^{R}=g_{i}^{R} E_{i, \circ}^{R}+\alpha_{i}^{L} E_{i, \circ}^{L}$ and substituting for $E_{i, \circ}^{R}$ and $E_{i, \circ}^{L}$ we get

$$
\begin{aligned}
E_{i}^{R}= & g_{i}^{R}\left(E_{i, \circ}^{X} \mathrm{e}^{\iota \delta}+E_{i, \circ}^{Y} \mathrm{e}^{-\iota \delta}\right) \\
& +\alpha_{i}^{L}\left(E_{i, \circ}^{X} \mathrm{e}^{-\iota \delta}+E_{i, \circ}^{Y} \mathrm{e}^{\iota \delta}\right) .
\end{aligned}
$$

Equation (3) for the case of correlation between a circularly polarized and a linearly polarized antenna, with polarization leakage in both the antennas, can be written as

$$
\begin{aligned}
X_{i j}^{R X}= & \left(g_{i}^{R} \mathrm{e}^{\iota \delta}+\alpha_{i}^{L} \mathrm{e}^{-\iota \delta}\right) g_{j}^{X^{\star}} \\
& +\left(g_{i}^{R} \mathrm{e}^{-\iota \delta}+\alpha_{i}^{L} \mathrm{e}^{\iota \delta}\right) \alpha_{j}^{Y^{\star}} \\
\equiv & g_{i}^{X^{\prime}} g_{j}^{X^{\star}}+\alpha_{i}^{Y^{\prime}} \alpha_{j}^{Y^{\star}}
\end{aligned}
$$

where $g_{i}^{X^{\prime}}=g_{i}^{R} \mathrm{e}^{\iota \delta}+\alpha_{i}^{L} \mathrm{e}^{-\iota \delta}$ and $\alpha_{i}^{Y^{\prime}}=g_{i}^{R} \mathrm{e}^{-\iota \delta}+\alpha_{i}^{L} \mathrm{e}^{\iota \delta}$. The leaky antsol solutions for the circularly polarized antenna in this case will correspond to $g_{i}^{X^{\prime}}$ and $\alpha_{i}^{Y^{\prime}}$.

Let $P_{i}=\alpha_{i}^{Y} / g_{i}^{X}\left(P_{i}=\alpha_{i}^{Y^{\prime}} / g_{i}^{X^{\prime}}\right.$ for the circularly polarized antenna). Then, the amplitude of $P_{i}$ is a measure

\footnotetext{
3 The VLA Calibrator manual is available on the web from http://www.aoc.nrao.edu/ gtaylor/calib.html
} 


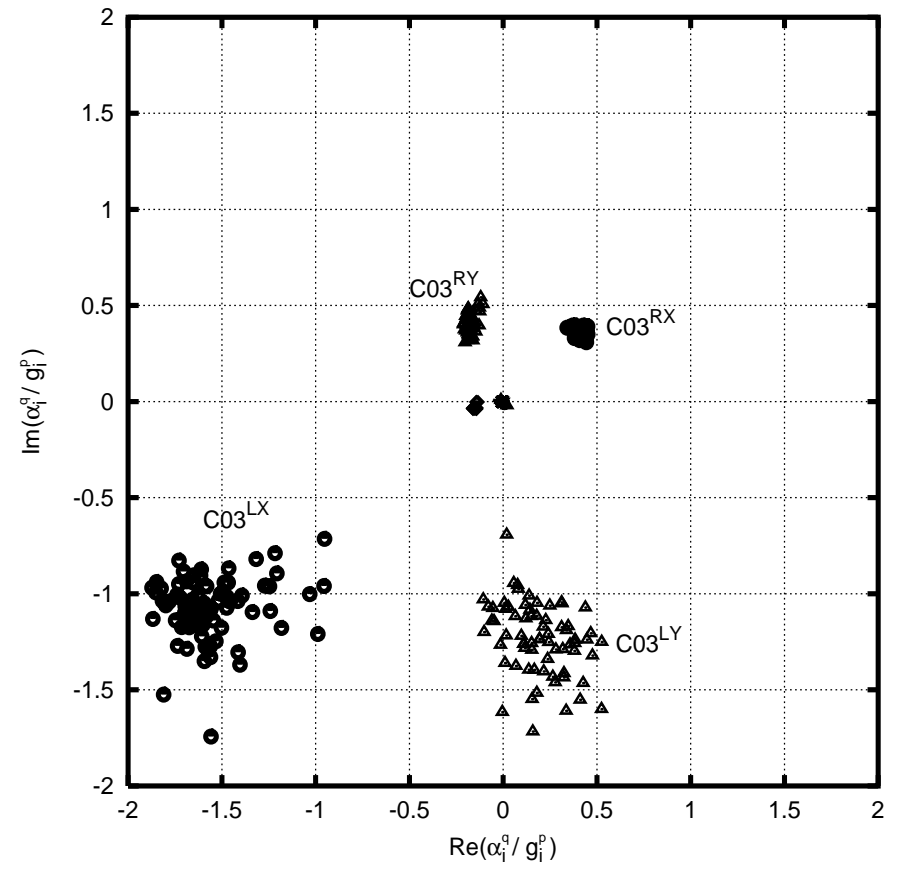

Fig. 3. Figure showing the results using visibilities with one circularly polarized antenna and all other linearly polarized antennas at $L$-band. The $x$ - and $y$-axis denote the real and imaginary parts of $\alpha_{i}^{q} / g_{i}^{p}$ respectively. $g_{i}^{p}$ and $\alpha_{i}^{q}$ were solved for every integration time $(\sim 17 \mathrm{~s})$. All linearly polarized antennas are close to the origin in this plot. The solutions for the circularly polarized antenna ( $\mathrm{C} 03)$ are the set of points away from the origin (shown by open circles and triangles). The two sets of points for this antenna, separated from each other by $\sim 180^{\circ}$ are solutions for the right- and left-circular polarization channel. The points denoted by open circles are from correlation between the right- and left-circular polarization of C03 with nominal linear $X$-polarization of the other antennas (labeled as $\mathrm{C} 03^{R X}$ and $\mathrm{C} 03^{L X}$ respectively). The points denoted by triangles are from correlation of $\mathrm{C} 03$ with nominal $Y$-polarization of the other antennas (labeled as $\mathrm{C} 03^{R Y}$ and $\left.\mathrm{C} 3^{L Y}\right)$.

of the fractional polarization leakage in the antenna while the phase of $P_{i}$ gives the phase difference between the signal from one of the feeds and the leaked signal from the other feed. For an ideal circularly polarized antenna, $\left|P_{i}\right| \approx 1$. A plot of the real and imaginary parts of this quantity for all antennas should therefore clearly show $P_{i}$ for the circularly polarized antenna with an amplitude of 1 and at an angle of $\pi / 2$ with respect to the nominal $X$-axis.

The real and imaginary parts of $P_{i}$ for all antennas from this experiment are shown in Fig. 3. The solutions were computed for every integration cycle of $\sim 17 \mathrm{~s}$ and the points on this plot represent the tip of phasor $P_{i}$. The collection of points near the origin are for all the linearly polarized antennas while the collection of two sets of points away from the origin, approximately at an angle of $\pi$ from each other, are for the circularly polarized antenna. The solutions found by leaky antsol match the expected results quite well. This therefore constitutes a reasonably controlled test with real data showing that the solutions indeed provide information about the polarization leakage in the system.

This experiment however provides much more information about the polarization properties of the antenna feeds used. The collection of points in the first quadrant denoted by open circles are the values of $P_{i}$ derived from the correlation between the nominal right-circularly polarized signal and the linearly polarized signals along the nominal $X$-axis from all other antennas. Points in the third quadrant are similarly derived using the left-circular signals. The set of points denoted by triangles in the second and fourth quadrant are derived using correlations of rightand left-circularly polarized signals with the linearly polarized signals along the nominal $Y$-axis from all other antennas.

A larger spread in the solutions using the left-circularly polarized signals indicates that the closure noise (from other unknown sources) in these signals is higher. The fact that the amplitude of $P_{i}$ derived using the right-circularly polarized signals is $\approx 0.5$ indicates that the nominal circularly polarized feed is in fact elliptically polarized with this axial ratio. The spread of $\pm 1-2 \%$ about the origin is indicative of polarization leakage at the level of few percent in the linearly polarized antennas as well. The leakage in one of the linearly polarized antennas is significantly larger $(\approx 4 \%)$. Since this kind of data is routinely taken on primary calibrators during GMRT observations for synthesis imaging, leaky antsol provides a useful diagnostic of system health, polarization performance and numbers needed to correct the data in high accuracy work.

The following test was also carried out to check that the closure phase on a triangle involving the circular feed was indeed mainly due to polarization effects. The three baselines making up this triangle were flagged as bad baselines from the input data and a new solution found for the gains and leakages of all antennas. This solution predicted the same closure phase (to within errors) as actually observed.

\section{Closure phase and the Poincaré sphere}

In this section we use right- and left-circular polarization states as the basis. A general elliptically polarized state can be written as a superposition of two states represented by the vector $\left(\begin{array}{l}\cos \theta / 2 \\ \sin \theta / 2 \mathrm{e}^{\iota \phi}\end{array}\right)$. Clearly, $\theta=\pi / 2$ corresponds to linear polarization and $\theta \neq 0, \pi / 2$ to elliptical polarization. Increasing $\phi$ by $\zeta$ rotates the direction of the linear state or the major axis of the ellipse by $\zeta / 2$. We can chose the phase of the basis so that $\phi=0$ corresponding to linear polarization along the $x$-axis. The Poincaré sphere representation of the state of polarization maps the general elliptic state to the point $(\theta, \phi)$ on the sphere. The properties of this representation are reviewed by Ramachandran \& Ramaseshan (1961). We are concerned here with one remarkable property, discovered by Pancharatnam $(1956,1975)$. Whenever there is constructive interference between two sources of radiation, 
it is natural to regard them as in phase. An unexpected property of this simple definition manifests itself when we consider 3 sources of radiation of different polarization that if a source $\mathrm{A}$ is in phase with $\mathrm{B}$ and $\mathrm{B}$ in phase with $\mathrm{C}, \mathrm{C}$ in general need not be in phase with $\mathrm{A}$. The phase difference between $\mathrm{A}$ and $\mathrm{C}$ is known in the optics literature as the geometric or Pancharatanam phase (see also Ramaseshan \& Nityananda 1986; Berry 1987). We show that this naturally occurs in radio interferometry of an unpolarized source with three antennas of different polarizations.

Let the polarization states of the three antennas be represented by $\left(\begin{array}{c}g_{1} \\ \alpha_{1}\end{array}\right),\left(\begin{array}{c}g_{2} \\ \alpha_{2}\end{array}\right)$, and $\left(\begin{array}{c}g_{3} \\ \alpha_{3}\end{array}\right)$ in a circular basis. Denoting the vector $\left(\begin{array}{c}g_{i} \\ \alpha_{i}\end{array}\right)$ by $\psi_{i}$, one clearly see that the visibility on the $1-2$ baseline is proportional to $\psi_{1}^{\dagger} \psi_{2}$. Hence the closure phase around a triangle made by antennas 1,2 , and 3 is the phase of the complex number (also called the triple product) $V_{123}=$ $\left(\psi_{1}^{\dagger} \psi_{2}\right)\left(\psi_{2}^{\dagger} \psi_{3}\right)\left(\psi_{3}^{\dagger} \psi_{1}\right)$. In the quantum mechanical literature, this type of quantity goes by the name of Bargmann's invariant and its connection to the geometric phase was made clear by Samuel \& Bhandari (1988). With some work, one can give a general proof that the closure phase (phase of $V_{123}$ ) is equal to half the solid angle subtended at the centre of the Poincare sphere by the points represented by $\psi_{1}, \psi_{2}$, and $\psi_{3}$ on the surface of the sphere. For the case where the polarization state of the three antennas are same, this phase is zero in general. However, when the polarization states of the antennas are different, this phase is non-zero.

The well known result that an arbitrary polarization state can be represented as a superposition of two orthogonal polarization states translates to representing any point on the Poincaré sphere by the superposition of two diametrically opposite states on a great circle passing through that point. For example, circular polarization can be expressed by two linear polarizations, each with intensity $1 / \sqrt{2}$. In the context of the present work, the nominally circularly polarized antenna maps to a point away from the equator on the Poincaré sphere (it would be exactly on the pole if it is purely circular) while the rest of the antennas map close to the equator (they would be exactly on the equator if they are purely linear and map to a single point if they were also identical). The visibility phase due to the extra baseline based term in Eq. (3) due to polarization mis-match is a consequence of the Pancharatanam phase mentioned above. This phase, on a triangle involving the circularly polarized antenna, will be close to the angle between the two linear antennas. For example, if $\psi_{1}=\left(\begin{array}{l}1 \\ \iota\end{array}\right), \psi_{2}=\left(\begin{array}{l}1 \\ 0\end{array}\right)$, and $\psi_{3}=\left(\begin{array}{c}\cos \gamma \\ \sin \gamma\end{array}\right)$, the phase of $V_{123}$ will be $\gamma$. This picture can be depicted by plotting the real and imaginary parts of $\alpha_{i}^{q} / g_{i}^{p}$, which is done in Fig. 3 . The circularly polarized antenna can be clearly located in this figure as the set of point away from the origin while the linearly polarized antennas as the set of points close to the origin. The collection of points located away but almost symmetrically about the origin represents the nominal right- and left-circularly polarized feeds. Points on the equator, but significantly away from the origin represents an imperfect linearly polarized antenna. Note that the average closure phase between the nominally linear antennas is close to zero, which defines the mean reference frame in Fig. 3.

\section{Conclusions}

Rogers (1983) pointed out that non ideal feed polarizations of the individual antennas of a radio interferometer can result into closure errors in the co-polar visibilities. In this paper we describe and demonstrate a method to measure the polarization leakage of individual antennas using the nominally co-polar visibilities for an unpolarized calibrator. This method can therefore be used as a useful tool for studying the polarization purity of the antennas of radio interferometers from the observations of unpolarized calibrators. However, since only unpolarized calibrators are used, the actual solution for the leakage parameters is subject to a degeneracy. This degeneracy does not affect the correction of the visibilities and can be used to remove the closure errors due to polarization leakage. Massi et al. (1997) have shown that such polarization leakage induced closure errors in the data from the EVN is the dominant effect of instrumental polarization. For the EVN, this effect can be seen as a reduction in the dynamic range of the images. Our method can be used for such data to remove these closure errors for unpolarized sources.

The general elliptic state of the polarization of radiation can be represented by a point on the Poincaré sphere. The phase difference between three coherent sources of radiation but with different states of polarization goes by the name of Pancharatanam or geometric phase in the optics literature. We interpret the co-polar visibilities with polarization leakages on the Poincaré sphere and show that the polarization induced closure phase errors in radio interferometers is same as the Pancharatanam phase of optics. The antenna based leakages also map to points on the Poincaré sphere and the ambiguity in the solution can be understood as a rigid rotation of the Poincaré sphere, which leaves the leakage solutions unchanged relative to each other.

Acknowledgements. We thank A. Omar and S. Roy who suggested that there could be closure errors in the data due to polarization leakage. Many useful discussions on this with A. P. Rao and Divya Oberoi are thankfully acknowledged. We also thank the telescope operators and other GMRT staff for their help and cooperation in making possible the observations used for this work. The GMRT is run by the National Centre for Radio Astrophysics of the Tata Institute of Fundamental Research.

This research has made use of NASA's Astrophysics Data System Abstract Service. All of this work was done using computers running the GNU/Linux operating system and it is a pleasure to thank all the numerous contributors to this software. 


\section{Appendix A: Non-uniqueness of solutions}

We discuss the non-uniqueness of the solutions of Eq. (3), and possible convenient conventions for choosing a specific solution. One obvious degeneracy is that multiplication of all the $\alpha$ 's by one common phase factor independent of antenna, and all the $g$ 's, by another, in general different, common factor, does not affect the right hand side of Eq. (3). Also, the equation was written in a specific basis, say right and left circular. But it would have had the same form when using any other orthogonal pair as basis since the source is unpolarized. Hence we are free to apply this change of basis to one solution to get another solution of Eq. (3). Under such a change, the coefficients transform according to

$$
\left(\begin{array}{c}
g^{\prime} \\
\alpha^{\prime}
\end{array}\right)=\left(\begin{array}{cc}
\cos \phi & \mathrm{e}^{\iota \gamma} \sin \phi \\
-\mathrm{e}^{-\iota \gamma} \sin \phi & \cos \phi
\end{array}\right)\left(\begin{array}{ll}
\mathrm{e}^{\iota \zeta_{1}} & 0 \\
0 & \mathrm{e}^{\iota \zeta_{2}}
\end{array}\right)\left(\begin{array}{c}
g \\
\alpha
\end{array}\right) .
$$

It is easy to verify that under this change, $\alpha_{i}^{\prime} \alpha_{j}^{\star \prime}+g_{i}^{\prime} g_{j}^{\star \prime}=\alpha_{i} \alpha_{j}^{\star}+g_{i} g_{j}^{\star}$. Clearly, since $\chi^{2}$ is unchanged by these transformations, an iterative algorithm will simply pick one member of the set of possible solutions, determined by the initial conditions. Having found one such, one could apply a suitable transformation to obtain a solution satisfying some desired condition. For example, if one has nominally linear feeds, one might impose the statistical condition that there is some mean linear basis with respect to which the leakage coefficients will be as small as possible. Such a condition has the advantage that a perfect set of feeds is not described in a roundabout way as a set of leaky feeds with identical coefficients, simply because the basis chosen was different. Carrying out the minimization of $\sum\left|g_{i}\right|^{2}$ by the method of Lagrange multipliers, subject to a constant $\chi^{2}$, we obtain the condition that $\sum \alpha_{i}^{*} g_{i}=0$. This solution can be interpreted as requiring the leakage coefficients to be orthogonal to the gains, and is reasonable when we think about the opposite kind of situation, when the leakages are "parallel" to the gains, i.e. identical apart from a multiplicative constant. In such a case, we would obviously change the basis to make the new leakage zero. If we have a solution which does not satisfy this orthogonality condition, we can bring it about in two steps. First, choose an overall phase for the $\alpha$ 's so that $\sum \alpha_{i}^{\star} g_{i}$ is real. Then, carry out a rotation in the $g-\alpha$ plane by an angle $\theta$ satisfying $\tan \theta=\sum \alpha_{i}^{*} g_{i} /\left(\sum\left(g_{i} g_{i}^{\star}-\alpha_{i} \alpha_{i}^{\star}\right)\right.$. This rotation has been so chosen that it makes the leakage "orthogonal" to the gains, in the sense required above. Even after this is done, we still have the freedom to define the phase zero independently for the two orthogonal states. This is because we are only dealing with unpolarized sources.
Of course, if we had a linearly polarized calibrator, the relative phase of right and left circular signals would not be arbitrary.

A more geometric view of this degeneracy is obtained when we think in terms of the Poincaré sphere representation of the states of polarization of all the feeds. The cross correlation between the outputs of two feeds, both of which receive unpolarized radiation, has a magnitude equal to the cosine of half the angle between the representative points on the sphere. Measurements of all such cross correlations with unpolarized radiation fixes the relative geometry of the points on the sphere, while leaving a two parameter degeneracy corresponding to overall rigid rotations of the sphere. This degeneracy can be lifted by the measurement of one polarized source at many parallactic angles.

Finally, we note that for the purpose of correcting the observations of unpolarized sources for the effects of nonidentical feed polarization, the degeneracy is unimportant, because the correction factor is precisely the right hand side of Eq. (3) which is unaffected by all the transformations we have discussed.

\section{References}

Berry, M. V. 1987, J. Mod. Opt., 34, 1401

Bhatnagar, S. 1999, Computation Of Antenna Dependent Complex Gains, Tech. rep., National Centre for Radio Astrophysics, Pune

Hamaker, J. P., Bregman, J. D., \& Sault, R. J. 1996, A\&AS, 117,137

Massi, M., \& Aaron, S. 1997a, EVN Tech. Memo, N75

Massi, M., \& Aaron, S. 1997b, EVN Tech. Memo, N77

Massi, M., Rioja, M., Gabuzda, D., et al. 1997, A\&A, 318, L32

Massi, M., Ruf, K., \& Orfei, A. 1998, EVN Tech. Memo, N85

Palka, B. P. 1990, Complex Functional Analysis (SpringerVerlag)

Pancharatnam, S. 1956, S. Proc. Indian Aad. Sci., A44, 247

Pancharatnam, S. 1975, Collected Works of S. Pancharatnam (Oxford Univ. Press), 77

Ramachandran, G. N., \& Ramaseshan, S. 1961, Handbuch der Physik, vol. 25, part 1 (Springer, Berlin)

Ramaseshan, S., \& Nityananda, R. 1986, Current Sci., 55, 1125

Rogers, A. E. E. 1983, VLB Array Memo No. 253

Samuel, J., \& Bhandari, R. 1988, Phys. Rev. Lett., 60, 2339

Sault, R. J., Hamaker, J. P., \& Bregman, J. D. 1996, A\&AS, 117,149

Thompson, A. R., \& D'Addario, L. R. 1982, Radio Sci., 17, 357

Thompson, A. R., Moran, J. M., \& Swenson, G. W. J. 1986, Interferometry and Synthesis in Radio Astronomy (John Wiley \& Sons, Inc.) 